\title{
Research Article \\ Synthetic Jet Actuator-Based Aircraft Tracking Using a Continuous Robust Nonlinear Control Strategy
}

\author{
N. Ramos-Pedroza, ${ }^{1}$ W. MacKunis, ${ }^{1}$ and M. Reyhanoglu ${ }^{2}$ \\ ${ }^{1}$ Department of Physical Sciences, Embry-Riddle Aeronautical University, Daytona Beach, FL 32114, USA \\ ${ }^{2}$ Department of Engineering, University of North Carolina at Asheville, Asheville, NC 28804, USA \\ Correspondence should be addressed to M. Reyhanoglu; reyhanoglu@unca.edu
}

Received 16 April 2017; Revised 18 July 2017; Accepted 3 October 2017; Published 20 December 2017

Academic Editor: Christopher J. Damaren

Copyright ( 2017 N. Ramos-Pedroza et al. This is an open access article distributed under the Creative Commons Attribution License, which permits unrestricted use, distribution, and reproduction in any medium, provided the original work is properly cited.

\begin{abstract}
A robust nonlinear control law that achieves trajectory tracking control for unmanned aerial vehicles (UAVs) equipped with synthetic jet actuators (SJAs) is presented in this paper. A key challenge in the control design is that the dynamic characteristics of SJAs are nonlinear and contain parametric uncertainty. The challenge resulting from the uncertain SJA actuator parameters is mitigated via innovative algebraic manipulation in the tracking error system derivation along with a robust nonlinear control law employing constant SJA parameter estimates. A key contribution of the paper is a rigorous analysis of the range of SJA actuator parameter uncertainty within which asymptotic UAV trajectory tracking can be achieved. A rigorous stability analysis is carried out to prove semiglobal asymptotic trajectory tracking. Detailed simulation results are included to illustrate the effectiveness of the proposed control law in the presence of wind gusts and varying levels of SJA actuator parameter uncertainty.
\end{abstract}

\section{Introduction}

The recent surge of interest in applications involving UAVs has motivated the development of low-mass actuators with reduced power requirements. Based on this, the use of SJAs has emerged as a popular tool for UAV control applications. SJAs can be used in a variety of applications, including trajectory tracking control, limit cycle oscillation (LCO) suppression, and boundary-layer flow control. The operation of SJAs is based on an effective combination of electrical, mechanical, and acoustic components [1]. SJAs transfer linear momentum to a flow system through vibration-induced oscillation of fluid flow through a narrow opening (see Figure 1). The oscillations are created by a piezoelectric membrane that operates inside of an air-filled cavity. The oscillating air in the cavity generates fluid vortices (jets) that travel away from the orifice. Since the jets are created using only the air in the surrounding environment, SJAs do not require space for a fuel supply. Moreover, SJAs are capable of transferring momentum to the system through a zero- net mass injection of air across the boundary. These virtues make SJAs an attractive option in UAV applications.

Under the operating conditions characteristic of UAV flight, a laminar separation bubble can form near the boundary layer, and total separation can occur if the angle of attack (AoA) is high enough [2]. This decreases the efficiency (i.e., lift/drag characteristics) of the airfoil. By endowing the airfoil with surface-embedded SJAs, active separation control systems can be developed. Flow separation control can be achieved using SJAs by virtue of their ability to energize the boundary layer by adding or removing momentum to or from the boundary layer [3-5]. SJAs are also capable of decreasing drag by delaying the flow separation point in the airfoil boundary layer [6]. In addition, SJAs can expand the usable range of the AoA, improving aircraft maneuverability [7]. Arrays containing multiple SJAs can be utilized to achieve aircraft tracking control $[8,9]$. By using SJAs as replacements for mechanical control surfaces (e.g., elevators and ailerons), radar cross-section can be reduced, and UAV weight, cost, and mechanical complexity can also be reduced. 


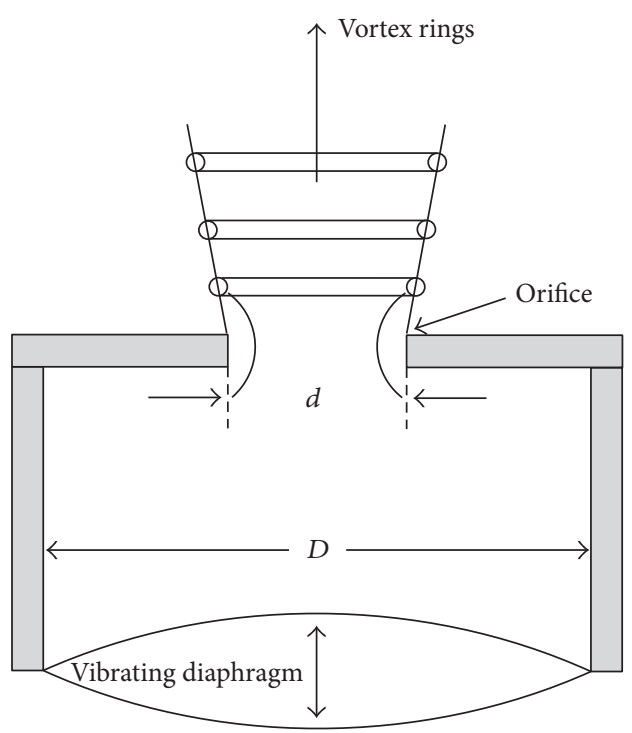

FIgURE 1: Schematic for a synthetic jet actuator.

The challenges in SJA-based control design stem from the fact that the input-output characteristics of SJAs are nonlinear and contain uncertain parameters (see Figure 2). In addition to the challenges involved in control design in the presence of SJA actuator uncertainty, control design for UAV in off-nominal operating conditions (e.g., wind gusts) creates further challenges. Various approaches have recently been developed for aircraft tracking control using SJAs (e.g., see $[8,10-13])$, where the SJA actuator uncertainty is compensated using adaptive control methods or neural networks. Other popular approaches for SJA-based control are computational fluid dynamics- (CFD-) based numerical techniques (see [14-28]). Adaptive control, neural networkbased control, and numerical CFD methods have been shown to be effective in their respective SJA-based control tasks. However, the focus of this paper is on the design and rigorous performance analysis of a computationally minimal nonlinear SJA-based control method, which can be implemented without adaptive parameter update laws, intelligent control techniques (e.g., neural networks or fuzzy logic rule sets), or heavy computations.

A robust nonlinear control method is presented in this paper that is proven to achieve asymptotic trajectory tracking control for a UAV in the presence of SJA actuator nonlinearity and parametric uncertainty in addition to unmodelled disturbances resulting from wind gusts. The challenge resulting from the uncertain SJA actuator parameters is mitigated through innovative algebraic manipulation in the tracking error system derivation along with a robust nonlinear control law employing constant "best guess" parameter estimates. A key contribution of the proposed control design is a rigorous analysis of the range of SJA actuator parameter uncertainty within which asymptotic UAV trajectory tracking can be achieved. Semiglobal asymptotic trajectory tracking is proven via a Lyapunov-based analysis, and detailed simulation results are provided to illustrate the performance of the proposed control law in the presence of wind gusts and varying levels of SJA actuator parameter uncertainty. A preliminary version of this result was published in the 2013 IEEE Conference on Decision and Control (CDC), but the current result includes the following additions and extensions beyond the CDC result: (1) rigorous stability analysis that now provides a detailed derivation of the operational region within which asymptotic tracking can be proved; (2) a significant extension to the theoretical control law derivation, including the additions of Lemma 1, Property 1, Assumption 3, and Remark 4; (3) a significantly expanded numerical simulation results section, which now includes Monte Carlo-type simulation results of the closed-loop control system under 20 different sets of uncertain SJA parameters that deviate from nominal by up to $35 \%$; (4) the addition of an appendix, which includes a detailed derivation of the control gain conditions required to prove asymptotic stability (i.e., proof of Lemma 2).

\section{Dynamic Model and Properties}

The dynamic model being considered in this paper incorporates the effects of parametric uncertainty in the aircraft dynamics, along with unmodelled external disturbances, and the inherent SJA actuator nonlinearity and parametric uncertainty. Specifically, the aircraft dynamic model can be expressed as (see, e.g., $[5,8-10,13,29-32]$ )

$$
\dot{x}=\mathbf{A} x+\mathbf{B u}+f(x, t)
$$

where $\mathbf{A} \in \mathbb{R}^{n \times n}$ and $\mathbf{B} \in \mathbb{R}^{n \times m}$ denote the uncertain state and input matrices, respectively, and $f(x, t) \in \mathbb{R}^{n}$ represents an unmodelled norm-bounded disturbance. The disturbance term $f(x, t)$ could represent the effects of external disturbances, such as wind gusts, or model inaccuracies resulting from linearization, for example. In (1), the control input $\mathbf{u}(t) \triangleq\left[\mathbf{u}_{1}(t) \cdots \mathbf{u}_{m}(t)\right]^{T} \in \mathbb{R}^{m}$ represents the virtual surface deflections resulting from $m$ arrays of SJA. These virtual surface deflections help create the lift forces on the outer trailing edge of the array [13]. Figure 2 shows the virtual deflection angle versus voltage for four different values of the SJA parameter $\theta_{1}^{*}$. A well-accepted empirically determined model of the SJA's dynamics can be expressed as $[8,10,12,13]$

$$
\mathbf{u}_{i}=\theta_{2 i}^{*}-\frac{\theta_{1 i}^{*}}{v_{i}}, \quad i=1,2, \ldots, m
$$

where $v_{i}(t)=\mathbf{A}_{\mathrm{pp} i}^{2}(t) \in \mathbb{R}$ denotes the peak-to-peak voltage acting on the $i$ th SJA array and $\theta_{1 i}^{*}, \theta_{2 i}^{*} \in \mathbb{R}$ denote uncertain positive physical parameters. The expression in (2) illuminates the challenges inherent in SJA-based control design: The control inputs $\mathbf{u}_{i}(t)$ depend nonlinearly on the voltage control signal $v_{i}(t)$ and include the uncertain parameters $\theta_{1 i}^{*}$ and $\theta_{2 i}^{*}$. In the subsequent control development, these challenges will be mitigated through innovative algebraic manipulation in the tracking error system development along with a robust, continuous nonlinear control method.

By substituting (2) into (1), the SJA-based dynamic model can be expressed as 


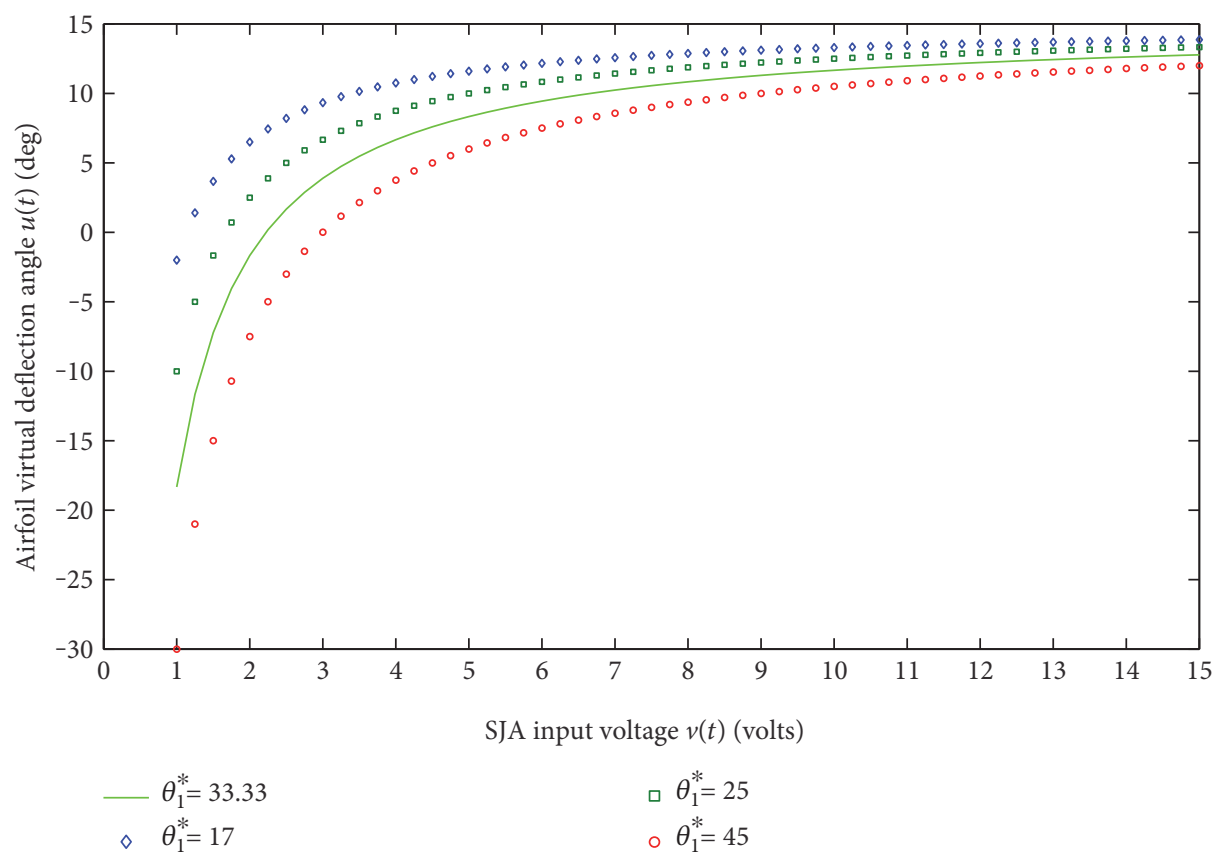

FIgURE 2: The virtual deflection angle versus control voltage for a SJA, showing the high degree of nonlinearity and the effect of parametric uncertainty in the SJA dynamic model. A well-accepted nominal value for the parameter is shown in green, and the three other plots show the SJA dynamic characteristics under off-nominal operating conditions.

$$
\dot{x}=\mathbf{A} x+\sum_{i=1}^{m} \mathbf{B}_{i} \mathbf{u}_{i}+f(x, t) .
$$

In $(3), \quad \mathbf{B}_{i} \triangleq\left[\mathbf{B}_{1 i} \cdots \mathbf{B}_{n i}\right]^{T} \in \mathbb{R}^{n} \forall i=1, \ldots, m$, where $\mathbf{B}_{i j}$ represents the $(i, j)$ th element of the uncertain $\mathbf{B}$ matrix.

Assumption 1. The disturbance $f(x, t)$ is sufficiently smooth in the sense that the first and second time derivatives $f(x, t)$ and $\ddot{f}(x, t)$ are bounded, provided that $x(t)$ is bounded.

2.1. Wind Gust Model. This section describes the details of the wind gust model (i.e., the disturbance term $f(x, t)$ introduced in (1)) that is being considered in this paper. The Federal Aviation Regulations (FAR) [33] describe a vertical wind gust as a bounded nonlinearity along the longitudinal axis as

$$
f(x, t)=\left[\begin{array}{c}
-11.1 \\
7.2 \\
37.4 \\
0
\end{array}\right] \frac{1}{V_{0}}\left\{\frac{U_{d s}}{2}\left[1-\cos \left(\frac{\pi s}{H}\right)\right]\right\}
$$

In (4), $H$ denotes the distance ( $\mathrm{m}$ ) along the airplane's flight path for the wind gust to reach its peak velocity, $V_{0}$ $(\mathrm{m} / \mathrm{s})$ is the forward velocity of the aircraft when it enters the gust, $s \in\left[\begin{array}{ll}0 & 2 H\end{array}\right]$ denotes the distance penetrated into the wind gust $(\mathrm{m})$, and $U_{d s}$ represents the design gust velocity $(\mathrm{m} / \mathrm{s})$. The wind gust model used in the subsequent numerical simulation results is based on the mathematical model in (4).
2.2. Robust Nonlinearity Inverse. The main contribution presented here is the mathematical development that demonstrates how a computationally inexpensive, robust nonlinear control method can be designed, which compensates for the parametric uncertainty and nonlinearity present in the SJA actuator dynamics. To achieve this, a robust-inverse control design structure will be utilized for the voltage control signal $v_{i}(t)$, which contains constant "best guess" estimates of the uncertain SJA parameters $\theta_{1 i}^{*}$ and $\theta_{2 i}^{*}$. The robust-inverse control structure is given by [9]

$$
v_{i}(t)=\frac{\widehat{\theta}_{1 i}}{\widehat{\theta}_{2 i}-\mathbf{u}_{d i}(t)}, \quad i=1, \ldots, m,
$$

where $\widehat{\theta}_{1 i}, \widehat{\theta}_{2 i} \in \mathbb{R}^{+}$are constant feedforward estimates of $\theta_{1 i}^{*}$ and $\theta_{2 i}^{*}$, respectively, and $\mathbf{u}_{d i}(t) \in \mathbb{R} \forall i=1, \ldots, m$ are subsequently defined auxiliary control terms.

Remark 1 (control structure). The robust-inverse control structure is one of the primary contributions of the proposed control design. In contrast to standard adaptive control methods to compensate for parametric actuator uncertainty, it is shown in the current result that this robust nonlinear control method compensates for a significantly higher level of uncertainty in the SJA parameters (see Simulation Results for details).

Remark 2 (avoiding singularities). Based on (5), singularities will occur when $\mathbf{u}_{d i}(t)=\widehat{\theta}_{2 i}$. To guarantee that the control law in (5) does not encounter these singularities, the auxiliary control terms $\mathbf{u}_{d i}(t)$ for $i=1,2, \ldots, m$ will incorporate following algorithm [29]: 


$$
\mathbf{u}_{d i}(t)= \begin{cases}\widehat{\theta}_{2 i}-\epsilon, & \text { if } g\left(\boldsymbol{\mu}_{0}(t), \boldsymbol{\mu}_{1}(t)\right) \geq \widehat{\theta}_{2 i}-\varepsilon, \\ g\left(\boldsymbol{\mu}_{0}(t), \boldsymbol{\mu}_{1}(t)\right), & \text { otherwise, }\end{cases}
$$

where $\varepsilon \in \mathbb{R}^{+}$is a user-defined design parameter, $g(\cdot)$ is a subsequently defined control input function, and $\boldsymbol{\mu}_{0}(t)$, $\boldsymbol{\mu}_{1}(t) \in \mathbb{R}^{m}$ are subsequently defined auxiliary control signals. Note that the control effectiveness can be ensured over an arbitrarily wide range of voltage input signals $v_{i}(t)$ through judicious selection of the design parameter $\varepsilon$.

In addition, singularities would be encountered in (2) when the voltage signal $v_{i}(t)=0$; however, this singular condition can be avoided by simply selecting $\widehat{\theta}_{1 i}>0$ for $i=1,2, \ldots, m$.

Remark 3. Numerical simulation results demonstrate that the control term $\mathbf{u}_{d i}(t)$ in (5) achieves asymptotic trajectory tracking and disturbance rejection in the presence of a significant deviation between the estimated and actual SJA parameter values $\widehat{\theta}_{j i}$ and $\theta_{j i}^{*}, j=1,2$.

2.3. Control Development. The objective is to ensure that the actual aircraft state $x(t)$ tracks a model reference (desired) state. Based on the mathematical structure of the dynamic model in (1), the model reference system is designed as

$$
\dot{x}_{m}=\mathbf{A}_{m} x_{m}+\mathbf{B}_{m} \delta,
$$

where $x_{m}(t) \in \mathbb{R}^{n}$ is the model reference state (i.e., the desired trajectory), $\mathbf{A}_{m} \in \mathbb{R}^{n \times n}$ denotes the model reference state matrix, $\mathbf{B}_{m} \in \mathbb{R}^{n}$ is the model reference input gain matrix, and $\delta(t) \in \mathbb{R}$ is the reference input (e.g., a pilot or autopilot command). The parameters of the reference model in (7) are selected such that the system achieves favorable flight performance characteristics in terms of convergence time and steady-state error, for example.

Assumption 2. The state of the model reference system remains bounded and sufficiently smooth in the sense that $x_{m}(t), \dot{x}_{m}(t), \ddot{x}_{m}(t), \dddot{x}_{m}(t) \in \mathscr{L}_{\infty} \forall t \geq 0$.

To quantify the control objective, a trajectory tracking error $\mathbf{e}(t) \in \mathbb{R}^{n}$ is defined as

$$
\mathbf{e}=x-x_{m}
$$

To facilitate the derivation of the error system dynamics, an auxiliary (filtered) error signal $r(t)$ is defined as

$$
\mathbf{r}=\dot{\mathbf{e}}+\gamma \mathbf{e},
$$

where $\gamma \in \mathbb{R}^{+}$is a constant control gain. By calculating the time derivative of (9) and substituting (1) and (8), the open-loop error system dynamics are obtained as

$$
\dot{\mathbf{r}}=\mathbf{A} \dot{\mathbf{e}}+\mathbf{A} \dot{x}_{m}+\sum_{i=1}^{m} \mathbf{B}_{i}\left(\frac{\theta_{1 i}^{*}}{\widehat{\theta}_{1 i}} \dot{\mathbf{u}}_{d i}(t)\right)+\dot{f}(x, t)-\ddot{x}_{m}+\gamma \dot{\mathbf{e}} .
$$

Remark 4. Although the constant portion of the SJA actuator model in (2) vanishes upon calculating the time derivative to obtain (10), the complete SJA model is incorporated in implementation by using (2) and (5). Thus, the subsequent simulation results incorporate the full SJA actuator model.

The open-loop error system in (10) can be rewritten in a more compact form as

$$
\dot{\mathbf{r}}=\tilde{\mathbf{N}}+\mathbf{N}_{d}+\mathbf{\Omega} \dot{\mathbf{u}}_{d}(t)-\mathbf{S e},
$$

where $\boldsymbol{\Omega} \in \mathbb{R}^{n \times m}$ is a constant uncertain matrix, $\mathbf{S} \in \mathbb{R}^{n \times n}$ is a subsequently defined auxiliary matrix, and $\mathbf{u}_{d}(t) \triangleq$ $\left[\mathbf{u}_{d 1}(t) \cdots \mathbf{u}_{d m}(t)\right]^{T} \in \mathbb{R}^{m}$ is the auxiliary control vector. In (11), the unknown, unmeasurable auxiliary terms $\tilde{\mathbf{N}}(t)$ and $\mathbf{N}_{d}(t)$ are explicitly defined as

$$
\begin{aligned}
& \tilde{\mathbf{N}} \triangleq \mathbf{A} \dot{\mathbf{e}}+\gamma \dot{\mathbf{e}}+\mathbf{S e}+\left(\dot{f}(x, t)-\dot{f}\left(x_{m}, t\right)\right), \\
& \mathbf{N}_{d} \triangleq \mathbf{A} \dot{x}_{m}-\ddot{x}_{m}+\dot{f}\left(x_{m}, t\right) .
\end{aligned}
$$

The motivation for the separation of terms as in (12) is based on the fact that the following bounding inequalities can be developed:

$$
\begin{aligned}
\|\tilde{\mathbf{N}}\| & \leq \rho(\|\mathbf{z}\|)\|\mathbf{z}\|, \\
\left\|\mathbf{N}_{d}\right\| & \leq \zeta_{\mathbf{N}_{d}}, \\
\left\|\dot{\mathbf{N}}_{d}\right\| & \leq \zeta_{\dot{\mathbf{N}}_{d}},
\end{aligned}
$$

where $\rho_{0}(\cdot) \in \mathbb{R}$ is a positive globally invertible nondecreasing function, $\zeta_{\mathbf{N}_{d}} \zeta_{\dot{N}_{d}} \in \mathbb{R}^{+}$are known bounding constants, and $\mathbf{z}(t) \in \mathbb{R}^{2 n}$ is an augmented tracking error vector that is defined as

$$
\mathbf{z} \triangleq\left[\mathbf{e}^{T} \mathbf{r}^{T}\right]^{T}
$$

2.4. Closed-Loop Error System. Based on the open-loop error dynamics in (11) and the subsequent stability analysis, the auxiliary control term $\mathbf{u}_{d}(t)$ is designed as

$$
\mathbf{u}_{d}(t)=\widehat{\boldsymbol{\Omega}}^{\#}\left(\boldsymbol{\mu}_{0}-\boldsymbol{\mu}_{1}\right),
$$

where $\widehat{\boldsymbol{\Omega}} \in \mathbb{R}^{n \times m}$ is a constant estimate of $\boldsymbol{\Omega}$ and $[\cdot]^{\#}$ denotes the matrix pseudoinverse. In (15), $\boldsymbol{\mu}_{0}(t), \boldsymbol{\mu}_{1}(t) \in \mathbb{R}^{n}$ denote feedback control terms defined as the generalized solutions to the differential equations

$$
\begin{aligned}
& \dot{\boldsymbol{\mu}}_{0}=-\left(\mathbf{k}_{s}+\mathbf{I}_{n \times n}\right) \mathbf{r}, \\
& \dot{\mu}_{1}=-\boldsymbol{\beta} \operatorname{sgn}(\mathbf{e}(t)),
\end{aligned}
$$

where $\beta, k_{s} \in \mathbb{R}^{n \times n}$ are constant, positive definite, diagonal control gain matrices.

Remark 5 (control input definitions). The motivation for defining the control terms $\boldsymbol{\mu}_{0}(t)$ and $\boldsymbol{\mu}_{1}(t)$ in terms of their time derivatives as in (16) is based on the subsequent Lyapunov-based stability analysis and the desire to design a continuous control law, which can be proven to achieve asymptotic rejection of norm-bounded disturbances. Note 
that integrating both sides of (16) results in a control expression that is continuous in time.

Remark 6 (integral signum term). Note that the auxiliary control term $\boldsymbol{\mu}_{1}(t)$ can be shown to be continuous by integrating both sides of the corresponding expression in (16). Mathematically, the integral of the signum of the tracking error $\mathbf{e}(t)$ can be interpreted as a finite-bandwidth signal (i.e., $\boldsymbol{\mu}_{1}(t)$ is a sawtooth wave with a finite slope). In practical implementation of the proposed control law, the control gain $\boldsymbol{\beta}$ in (16) can be tuned to adjust the slope of the sawtooth wave, thereby compensating for norm-bounded disturbances using high-frequency feedback (finite bandwidth), as opposed to the discontinuous (infinite bandwidth) high-gain feedback that is characteristic of standard sliding mode control methods (i.e., due to direct implementation of the $\operatorname{sgn}(\cdot)$ function in standard sliding mode control methods).

After substituting the time derivative of (15) into (11), the error dynamics can be expressed as

$$
\dot{\mathbf{r}}=\tilde{\mathbf{N}}+\mathbf{N}_{d}+\tilde{\mathbf{\Omega}}\left(\dot{\boldsymbol{\mu}}_{0}-\dot{\boldsymbol{\mu}}_{1}\right)-\mathbf{S e}
$$

where the constant uncertain matrix $\tilde{\Omega} \in \mathbb{R}^{n \times n}$ is defined as

$$
\tilde{\mathbf{\Omega}}=\mathbf{\Omega} \widehat{\mathbf{\Omega}}^{\#} .
$$

Lemma 1 [34]. Any positive definite matrix $\mathbf{X} \in \mathbb{R}^{n \times n}$ can be decomposed as

$$
\mathbf{X}=\mathbf{S T} \text {, }
$$

where $S \in \mathbb{R}^{n \times n}$ is a positive definite symmetric matrix and $T \in \mathbb{R}^{n \times n}$ is a unity upper triangular matrix.

Proof. Proof of Lemma 1 can be found in [34] and is omitted here for brevity.

Property 1. Since the matrix $S$ introduced in (19) is positive definite and symmetric, its inverse $\mathbf{S}^{-1}$ is also positive definite and symmetric. This property will be utilized in the subsequent stability analysis.

Assumption 3. Upper and lower bounds on the elements of the uncertain constant matrix $\Omega \in \mathbb{R}^{n \times n}$ are known such that the constant feed forward estimate $\widehat{\Omega} \in \mathbb{R}^{n \times n}$ can be chosen to render the product $\tilde{\boldsymbol{\Omega}}=\boldsymbol{\Omega} \widehat{\boldsymbol{\Omega}}^{-1}$ positive definite. Further, the estimate $\widehat{\Omega}$ is selected such that

$$
\tilde{\mathbf{\Omega}}=\mathbf{S T},
$$

where the unity upper triangular matrix $\mathbf{T}$ satisfies the diagonal dominance property

$$
\varepsilon \leq\left|\mathbf{T}_{i i}\right|-\sum_{k=i+1}^{n}\left|\mathbf{T}_{i k}\right| \leq Q, \quad i=1, \ldots, n-1,
$$

where $\varepsilon \in(0,1)$ and $Q \in \mathbb{R}^{+}$are known bounding constants and $\mathbf{T}_{i k} \in \mathbb{R}$ denotes the $(i, k)$ th element of the matrix $\mathbf{T}$. In
(20), the matrices $\mathbf{S}$ and $\mathbf{T}$ are defined in a manner similar to Lemma 1.

Remark 7. The subsequent numerical simulation results demonstrate that Assumption 3 is satisfied over a significant range of uncertainty between the estimated and actual values of the uncertain input-multiplicative matrix (i.e., deviations between $\widehat{\boldsymbol{\Omega}}$ and $\boldsymbol{\Omega}$ ). Specifically, the results show that asymptotic trajectory tracking is achieved when the constant estimates $\widehat{\theta}_{1 j}$ and $\widehat{\theta}_{2 j} \forall j=1, \ldots, m$ deviate from the actual values by more than $35 \%$.

After using the decomposition technique in (20), the open-loop error dynamics in (17) can be expressed as

$$
\mathbf{S}^{-1} \dot{r}=\tilde{\mathbf{N}}_{1}+\mathbf{N}_{d 1}+\mathbf{T}\left(\dot{\boldsymbol{\mu}}_{0}-\dot{\boldsymbol{\mu}}_{1}\right)-\mathbf{e}
$$

where

$$
\begin{gathered}
\tilde{\mathbf{N}}_{1} \triangleq \mathbf{S}^{-1} \tilde{\mathbf{N}}, \\
\mathbf{N}_{d 1} \triangleq \mathbf{S}^{-1} \mathbf{N}_{d} .
\end{gathered}
$$

Since $\mathbf{S}$ is positive definite, $\tilde{\mathbf{N}}_{1}(t)$ and $\mathbf{N}_{d 1}(t)$ satisfy the following inequalities:

$$
\begin{aligned}
& \left\|\tilde{\mathbf{N}}_{1}\right\| \leq \rho_{1}(\|\mathbf{z}\|)\|\mathbf{z}\|, \\
& \left\|\mathbf{N}_{d 1}\right\| \leq \zeta_{\mathbf{N}_{d 1}}, \\
& \left\|\dot{\mathbf{N}}_{d 1}\right\| \leq \zeta_{\dot{\mathbf{N}}_{d 1}},
\end{aligned}
$$

where $\rho_{1}(\cdot) \in \mathbb{R}$ is a positive, globally invertible nondecreasing function and $\zeta_{\mathbf{N}_{d 1}}, \zeta_{\dot{N}_{d 1}} \in \mathbb{R}^{+}$are known bounding constants. By using the fact that the uncertain matrix $\mathbf{T}$ is unity upper triangular, the error dynamics in (22) can be rewritten as

$$
\mathbf{S}^{-1} \dot{\mathbf{r}}=\tilde{\mathbf{N}}_{1}+\mathbf{N}_{d 1}+\dot{\boldsymbol{\mu}}_{0}+\overline{\mathbf{T}} \dot{\boldsymbol{\mu}}_{0}-\mathbf{T} \dot{\boldsymbol{\mu}}_{1}-\mathbf{e},
$$

where $\overline{\mathbf{T}} \triangleq \mathbf{T}-\mathbf{I}_{n \times n}$ is a strictly upper triangular matrix, and $\mathbf{I}_{n \times n}$ denotes the $n \times n$ identity matrix. After substituting the control expressions in (16), the closed-loop error system is obtained as

$$
\mathbf{S}^{-1} \dot{\mathbf{r}}=\tilde{\mathbf{N}}_{1}+\overline{\mathbf{T}} \dot{\boldsymbol{\mu}}_{0}+\mathbf{N}_{d 1}-\left(\mathbf{k}_{s}+\mathbf{I}_{n \times n}\right) \mathbf{r}-\mathbf{T} \dot{\boldsymbol{\mu}}_{1}-\mathbf{e} .
$$

After utilizing (16), the term $\overline{\mathbf{T}} \dot{\boldsymbol{\mu}}_{0}$ can be expressed as

$$
\overline{\mathbf{T}} \dot{\boldsymbol{\mu}}_{0}=\left[\begin{array}{c}
\sum_{j=2}^{n} \overline{\mathbf{T}}_{1 j} \dot{\boldsymbol{\mu}}_{0 j} \\
\sum_{j=3}^{n} \overline{\mathbf{T}}_{2 j} \dot{\boldsymbol{\mu}}_{0 j} \\
\vdots \\
\overline{\mathbf{T}}_{(n-1) n} \dot{\boldsymbol{\mu}}_{0 n} \\
0
\end{array}\right]=\left[\begin{array}{c}
\boldsymbol{\Lambda}_{\rho} \\
0
\end{array}\right],
$$


where the auxiliary signal $\boldsymbol{\Lambda}_{\rho} \triangleq\left[\boldsymbol{\Lambda}_{\rho 1}, \boldsymbol{\Lambda}_{\rho 2} \cdots \boldsymbol{\Lambda}_{\rho(n-1)}\right]^{T} \in \mathbb{R}^{n-1}$, with the individual elements defined as

$$
\Lambda_{\rho i} \triangleq-\sum_{j=i+1}^{n} \overline{\mathbf{T}}_{i j}\left(\mathbf{k}_{s j}+1\right) \mathbf{r}_{j}
$$

for $i=1, \ldots, n-1$ where the subscript $j$ indicates the $j$ th element of the vector. Based on the definitions in (16) and (27), $\Lambda_{\rho}$ can be upper bounded as

$$
\left\|\boldsymbol{\Lambda}_{\rho}\right\| \leq \rho_{\Lambda 1}\|\mathbf{z}\|
$$

where $\mathbf{z}(t)$ was previously defined in (14) and $\rho_{\Lambda 1} \in \mathbb{R}$ is a known positive bounding constant.

Remark 8. Note that based on (27) and (28), the bounding constant $\rho_{\Lambda 1}$ depends only on elements $i+1$ to $n$ of the control gain matrix $\mathbf{k}_{s}$ due to the strictly upper triangular nature of $\overline{\mathbf{T}}$. Thus, the element $\dot{\boldsymbol{\mu}}_{01}(t)$ of the control vector $\dot{\boldsymbol{\mu}}_{0}(t)$ does not appear in the term $\Lambda_{\rho}$. This fact will be utilized in the subsequent stability proof [9].

By utilizing (27), the error dynamics in (26) can be expressed as

$$
\mathbf{S}^{-1} \dot{\mathbf{r}}=\tilde{\mathbf{N}}_{2}+\mathbf{N}_{d 1}-\left(\mathbf{k}_{s}+\mathbf{I}_{n \times n}\right) \mathbf{r}-\mathbf{T} \dot{\boldsymbol{\mu}}_{1}-\mathbf{e},
$$

where

$$
\tilde{\mathbf{N}}_{2}=\tilde{\mathbf{N}}_{1}+\left[\begin{array}{c}
\Lambda_{\rho} \\
0
\end{array}\right]
$$

Based on (24), (29), and (31), $\tilde{\mathbf{N}}_{2}(t)$ satisfies the inequality

$$
\left\|\tilde{\mathbf{N}}_{2}\right\| \leq \rho_{2}(\|\mathbf{z}\|)\|\mathbf{z}\|,
$$

where $\rho_{2}(\cdot) \in \mathbb{R}$ is a positive, globally invertible nondecreasing function.

To facilitate the subsequent stability analysis, the control gain $\boldsymbol{\beta}$ introduced in (16) is selected to satisfy

$$
\boldsymbol{\beta}>\frac{1}{\varepsilon}\left(\zeta_{\mathrm{N}_{d 1}}+\frac{1}{\gamma} \zeta_{\mathbf{N}_{d 1}}\right),
$$

where $\zeta_{\mathbf{N}_{d 1}}$ and $\zeta_{\dot{\mathbf{N}}_{d 1}}$ are introduced in (24) and $\varepsilon$ is introduced in (21).

\section{Stability Analysis}

Let $\mathscr{D} \subset \mathbb{R}^{2 n+1}$ be a domain containing $w(t)=0$, where $w(t)$ $\in \mathbb{R}^{2 n+1}$ is defined as

$$
w(t) \triangleq\left[\mathbf{z}^{T}(t) \sqrt{P(t)}\right]^{T} .
$$

In (34), the auxiliary function $P(t) \in \mathbb{R}$ is defined as the generalized solution to the differential equation

$$
\dot{P}(t)=-L(t)
$$

$$
P(0)=\boldsymbol{\beta} Q|\mathbf{e}(0)|-\mathbf{e}^{T}(0) \mathbf{N}_{d 1}(0),
$$

where the auxiliary function $L(t) \in \mathbb{R}$ is defined as

$$
L(t)=\mathbf{r}^{T}\left(\mathbf{N}_{d 1}(t)-\mathbf{T} \dot{\boldsymbol{\mu}}_{1}\right) .
$$

Lemma 2. Provided the sufficient condition in (33) is satisfied, the following inequality can be obtained:

$$
\int_{0}^{t} L(\tau) d \tau \leq \boldsymbol{\beta} Q|\mathbf{e}(0)|-\mathbf{e}^{T}(0) \mathbf{N}_{d 1}(0) .
$$

Hence, (38) can be used to conclude that $P(t) \geq 0$.

Proof. Proof of Lemma 2 can be found in the appendix.

Theorem 1. The robust control law given by (5), (15), and (16) achieves asymptotic trajectory tracking in the sense that

$$
\|\mathbf{e}(t)\| \rightarrow 0, \quad \text { as } \quad t \rightarrow \infty,
$$

provided the control gain matrix $\mathbf{k}_{s}$ introduced in (16) is selected sufficiently large and $\boldsymbol{\beta}$ is selected to satisfy the sufficient condition in (33).

Proof. Let $V(w, t): \mathscr{D} \times[0, \infty) \rightarrow \mathbb{R}$ be a continuously differentiable, nonnegative function defined as

$$
V=\frac{1}{2} \mathbf{e}^{T} \mathbf{e}+\frac{1}{2} \mathbf{r}^{T} \mathbf{S}^{-1} \mathbf{r}+P,
$$

which satisfies the inequalities

$$
U_{1}(w) \leq V(w, t) \leq U_{2}(w),
$$

provided the sufficient condition in (33) is satisfied. In (41), the continuous positive definite functions $U_{1}(w), U_{2}(w) \in$ $\mathbb{R}$ are defined as

$$
\begin{aligned}
& U_{1}(w) \triangleq \eta_{1}\|w\|^{2}, \\
& U_{2}(w) \triangleq \eta_{2}\|w\|^{2},
\end{aligned}
$$

where $\eta_{1}, \eta_{2} \in \mathbb{R}$ are defined as

$$
\begin{aligned}
& \eta_{1} \triangleq \frac{1}{2} \min \left\{1, \lambda_{\min }\left(\mathbf{S}^{-1}\right)\right\}, \\
& \eta_{2} \triangleq \max \left\{\frac{1}{2} \lambda_{\max }\left(\mathbf{S}^{-1}\right), 1\right\},
\end{aligned}
$$

where $\lambda_{\min }(\cdot), \lambda_{\max }(\cdot)$ denote the minimum and maximum eigenvalues of the arguments, respectively. After taking the time derivative of (40), utilizing (9), (30), (35), and (37), and canceling common terms, $\dot{V}(t)$ can be expressed as

$$
\dot{V}=-\gamma\|\mathbf{e}\|^{2}-\|\mathbf{r}\|^{2}-\mathbf{r}^{T}\left(\tilde{\mathbf{N}}_{2}-\mathbf{k}_{s} \mathbf{r}\right)
$$

After using the upper bound for $\tilde{\mathbf{N}}_{2}(t)$ given in (32) and completing the squares for the parenthetic terms, $\dot{V}$ can be upper bounded as 


$$
\begin{aligned}
\dot{V} \leq & -\lambda_{0}\|\mathbf{z}\|^{2}+\frac{\rho_{2}(\|\mathbf{z}\|)^{2}}{4 \lambda_{\min }\left(\mathbf{k}_{s}\right)}\|\mathbf{z}\|^{2}-\lambda_{\min }\left(\mathbf{k}_{s}\right) \\
& \cdot\left(\|\mathbf{r}\|^{2}-\rho_{2}(\|\mathbf{z}\|)\|\mathbf{r}\|\|\mathbf{z}\|+\frac{\rho_{2}(\|\mathbf{z}\|)^{2}}{4 \lambda_{\min }^{2}\left(\mathbf{k}_{s}\right)}\|\mathbf{z}\|^{2}\right),
\end{aligned}
$$

where $\lambda_{0} \triangleq \min \{\gamma, 1\}$ and $\lambda_{\min }(\cdot)$ denotes the minimum eigenvalue of the argument. The upper bound in (45) can be rewritten as

$$
\dot{V} \leq-\left(\lambda_{0}-\frac{\rho_{2}(\|\mathbf{z}\|)^{2}}{4 \lambda_{\min }\left(\mathbf{k}_{s}\right)}\right)\|\mathbf{z}\|^{2}
$$

The following expression can be obtained from (46):

$$
\dot{V} \leq-U(w)
$$

where $U(w)=c\|\mathbf{z}\|^{2}$, for some positive constant $c \in \mathbb{R}$ is a continuous positive semidefinite function that is defined on the domain

$$
\mathscr{D} \triangleq\left\{w(t) \in \mathbb{R}^{2 n+1} \mid\|w\| \leq \rho_{2}^{-1}\left(2 \sqrt{\lambda_{\min }\left(\mathbf{k}_{s}\right) \lambda_{0}}\right)\right\} .
$$

The expressions (41) and (46) can be used to prove that $\mathbf{e}(t), \mathbf{r}(t) \in \mathscr{L}_{\infty}$ in $\mathscr{D}$. Given that $\mathbf{e}(t), \mathbf{r}(t) \in \mathscr{L}_{\infty}$, (9) can be used to show that $\dot{\mathbf{e}}(t) \in \mathscr{L}_{\infty}$ in $\mathscr{D}$. Given that $\mathbf{e}(t), \dot{\mathbf{e}}(t) \epsilon$ $\mathscr{L}_{\infty},(8)$ can be used along with Assumption 2 to prove that $x(t), \dot{x}(t) \in \mathscr{L}_{\infty}$ in $\mathscr{D}$. Based on the fact that $x(t) \in \mathscr{L}_{\infty}$, Assumption 1 can be utilized to show that $f(x, t) \in \mathscr{L}_{\infty}$ in $\mathscr{D}$. Since $x(t), \dot{x}(t), f(x, t) \in \mathscr{L}_{\infty},(1)$ can be used to show that $\mathbf{u}(t) \in \mathscr{L}_{\infty}$ in $\mathscr{D}$. Since $\mathbf{e}(t), \mathbf{r}(t) \in \mathscr{L}_{\infty}$, the expressions in (16) can be used to show that $\dot{\boldsymbol{\mu}}_{0}(t), \dot{\boldsymbol{\mu}}_{1}(t) \in \mathscr{L}_{\infty}$ in $\mathscr{D}$. Given that $\mathbf{e}(t), \mathbf{r}(t), \dot{\mu}_{1}(t) \in \mathscr{L}_{\infty}$, (30) can be used along with (32) to show that $\dot{\mathbf{r}}(t) \in \mathscr{L}_{\infty}$ in $\mathscr{D}$. Since $\dot{\mathbf{e}}(t), \dot{\mathbf{r}}(t) \epsilon$ $\mathscr{L}_{\infty}$ can be used to show that $\mathbf{e}(t)$ and $\mathbf{r}(t)$ are uniformly continuous in $\mathscr{D}$, thus, $\mathbf{z}(t)$ is uniformly continuous throughout the closed-loop controller operation. Hence, $U$ $(w)$ and $\mathbf{z}(t)$ can be used to prove that $U(w)$ is uniformly continuous in $\mathscr{D}$.

Let $\delta \subset \mathscr{D}$ denote a set defined as follows:

$$
\mathcal{S} \triangleq\left\{w(t) \subset \mathscr{D} \mid U(w(t)) \leq \eta_{1}\left(\rho_{2}^{-1}\left(2 \sqrt{\lambda_{\min }\left(\mathbf{k}_{s}\right) \lambda_{0}}\right)\right)^{2}\right\} .
$$

Theorem 8.4 of [35] can now be invoked to state that

$$
c\|\mathbf{z}(t)\|^{2} \rightarrow 0 \quad \text { as } \quad t \rightarrow \infty, \quad \forall w\left(t_{0}\right) \in \mathcal{S} .
$$

Based on the definition of $\mathbf{z}(t)$, (50) can be used to show that

$$
\|\mathbf{e}(t)\| \rightarrow 0 \quad \text { as } \quad t \rightarrow \infty, \quad \forall w\left(t_{0}\right) \in \mathcal{S} .
$$

Thus, asymptotic regulation of the pitching and plunging displacements can be achieved, provided the initial conditions are within the set $\mathcal{S}$, where $\mathcal{S}$ can be made arbitrarily large by increasing the control gain $\mathbf{k}_{s}$. Hence, this is a semiglobal asymptotic result.

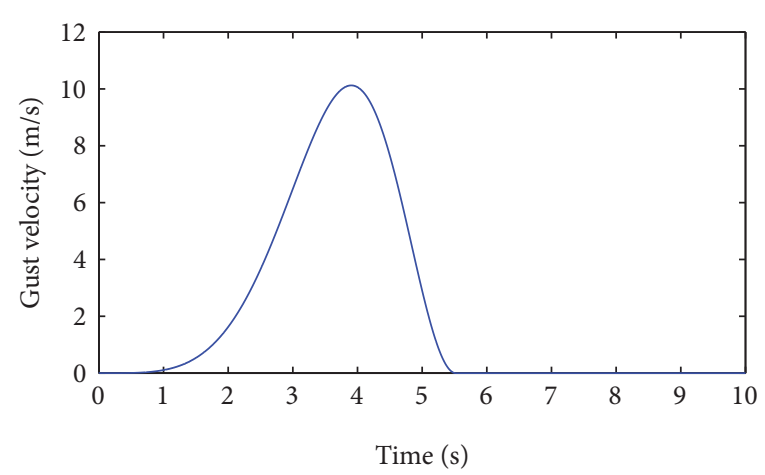

FIgURe 3: Vertical gust velocity.

\section{Simulation Results}

A numerical simulation was created to test the performance of the control design in (2), (5), (15), and (16). The simulation is based on the dynamic model in (1) and (2), where $n$ $=3$ and $m=6$ (i.e., 3-DOF flight control using 6 SJA arrays). The state vector contains the roll, pitch, and yaw rates, and the tracking error vector can be expressed as

$$
\mathbf{e}(t)=\left[\mathbf{e}_{1}(t), \mathbf{e}_{2}(t), \mathbf{e}_{3}(t)\right]^{T} .
$$

The state and input matrices, $\mathbf{A}$ and $\mathbf{B}$, and reference state and reference input matrices, $\mathbf{A}_{m}$ and $\mathbf{B}_{m}$, are defined based on the Barron Associates nonlinear tailless aircraft model (BANTAM) (for further details of the simulation model, see [8]). The 3-DOF linearized model for the BANTAM was obtained analytically during trim conditions, where $M=0.455$ is the Mach number, $\alpha=2.7:(\mathrm{deg})$ is angle of attack, and $\boldsymbol{\beta}_{s}=0:(\mathrm{deg})$ denotes the side slip angle. The simulation includes the effects of a wind gust in (4) as described in [33] at a velocity of $U_{d s}=10.12:(\mathrm{m} / \mathrm{s}), H=$ 15.24: $(\mathrm{m})$, and $V_{0}=25:(\mathrm{m} / \mathrm{s})$.

The reference state and input matrices used in the simulation are explicitly defined as

$$
\begin{aligned}
& \mathbf{A}_{m}=\left[\begin{array}{ccc}
-61.1446 & 0 & -7.5238 \\
0 & -174.3473 & 0 \\
-7.1579 & 0 & -1.4007
\end{array}\right], \\
& \mathbf{B}_{m}=\left[\begin{array}{c}
-1.7517 \\
0 \\
0.3096
\end{array}\right] .
\end{aligned}
$$

The matrices are $\mathbf{A}_{m} \in \mathbb{R}^{3 \times 3}$ and $\mathbf{B}_{m} \in \mathbb{R}^{3}$. The model reference (desired) state $x_{m}(t)$ in the simulation represents the desired external body axis motion that is generated in response to a reference command of (see (7))

$$
\delta(t)=\sin (t)
$$

The matrices $\mathbf{A}$ and $\mathbf{B}$ were obtained analytically from the dimensional aerodynamic coefficients of the BANTAM [8]. These matrices are given by 

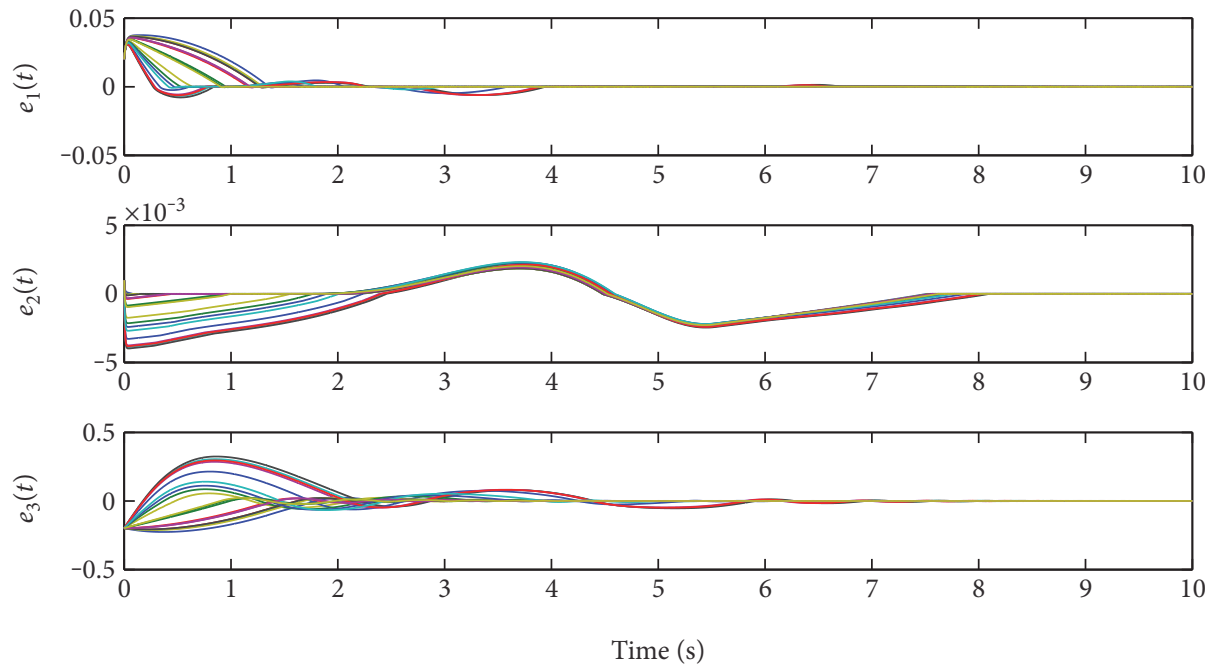

FIGURE 4: Closed-loop regulation of the steady state error.
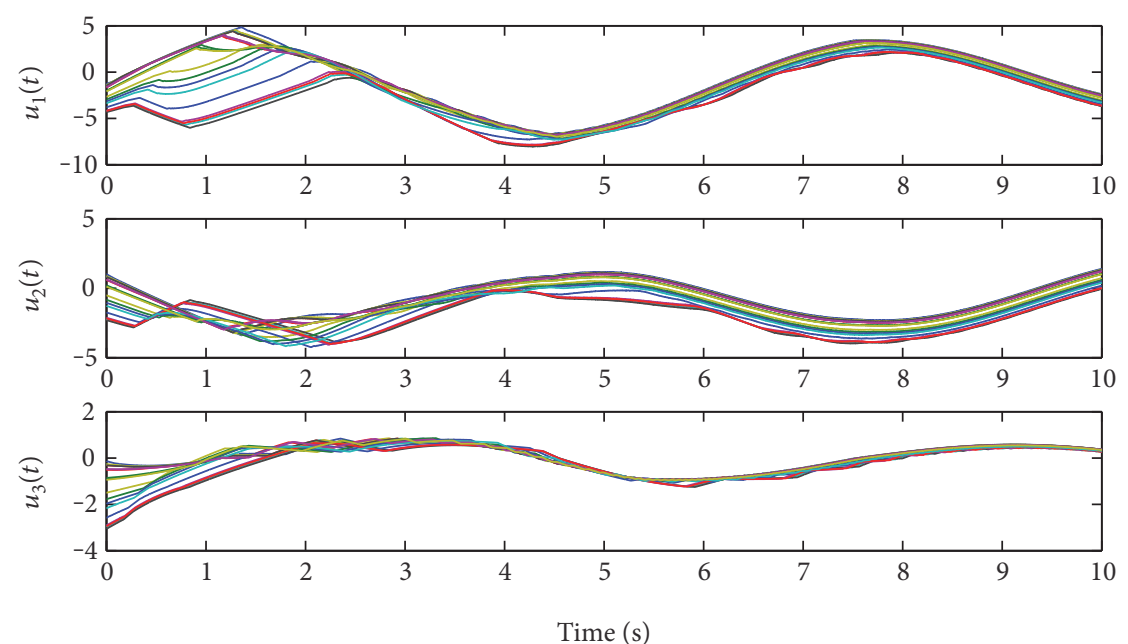

Figure 5: Virtual deflection angle control commands for the first three SJA arrays (i.e., $\mathbf{u}_{1}(t)$, $\mathbf{u}_{2}(t)$, and $\mathbf{u}_{3}(t)$ ) during closed-loop operation.
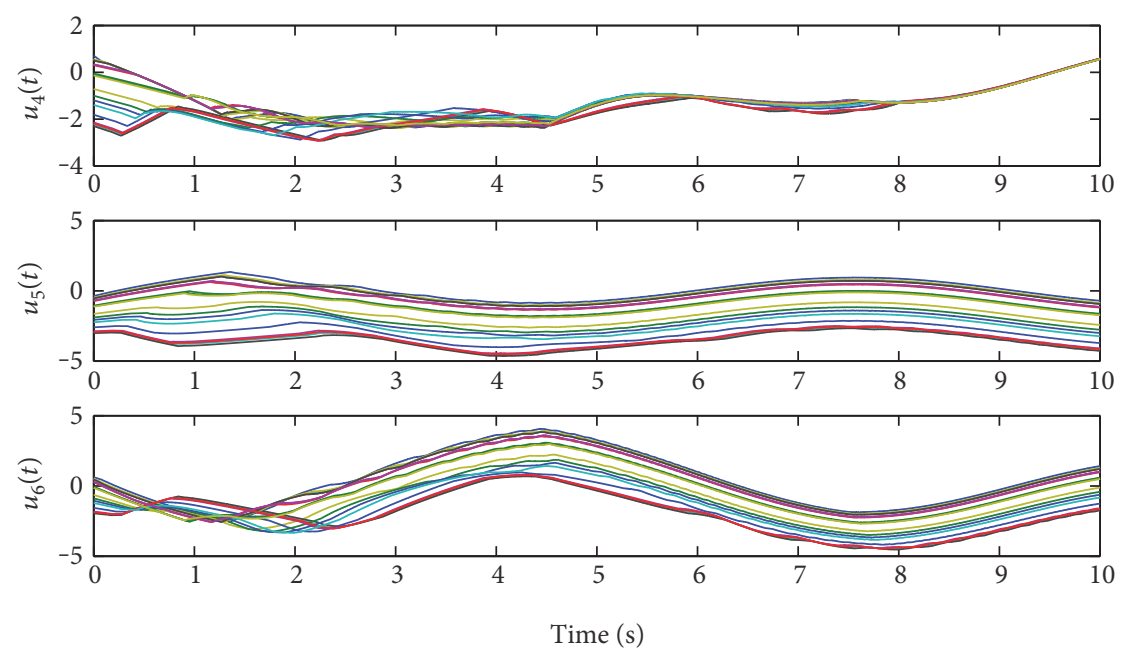

FIGURE 6: Virtual deflection angle control commands for the last three SJA arrays (i.e., $\mathbf{u}_{4}(t)$, $\mathbf{u}_{5}(t)$, and $\left.\mathbf{u}_{6}(t)\right)$ during closed-loop operation. 

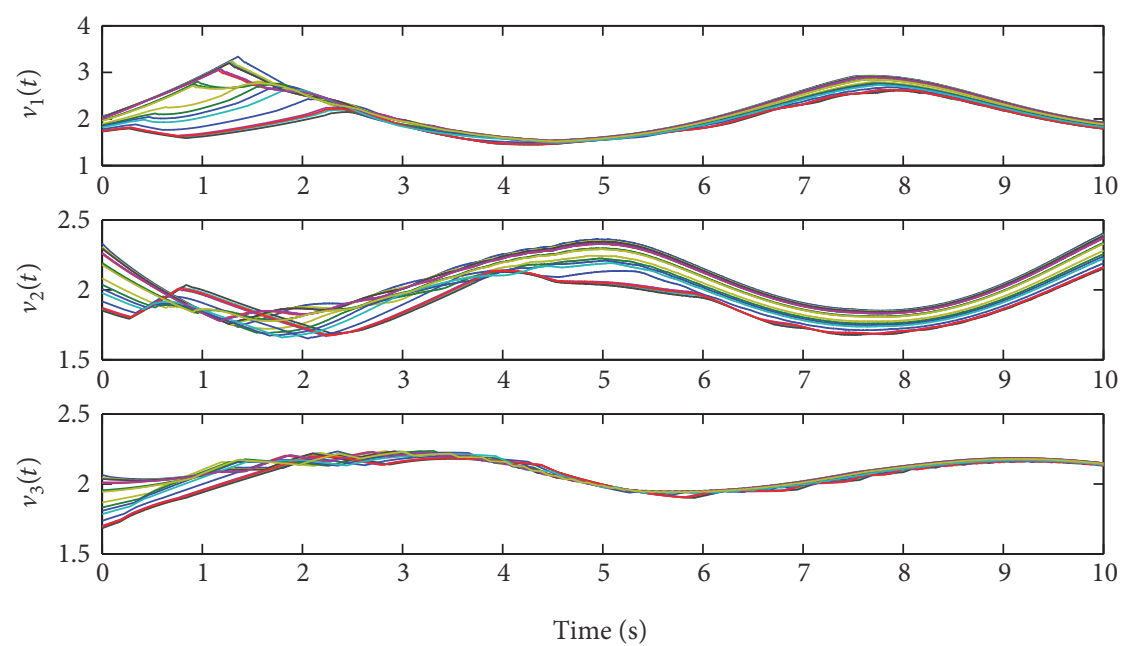

FIGURE 7: Control voltage signals commanded for the first three SJA arrays (i.e., $v_{1}(t), v_{2}(t)$, and $v_{3}(t)$ ) during closed-loop operation.
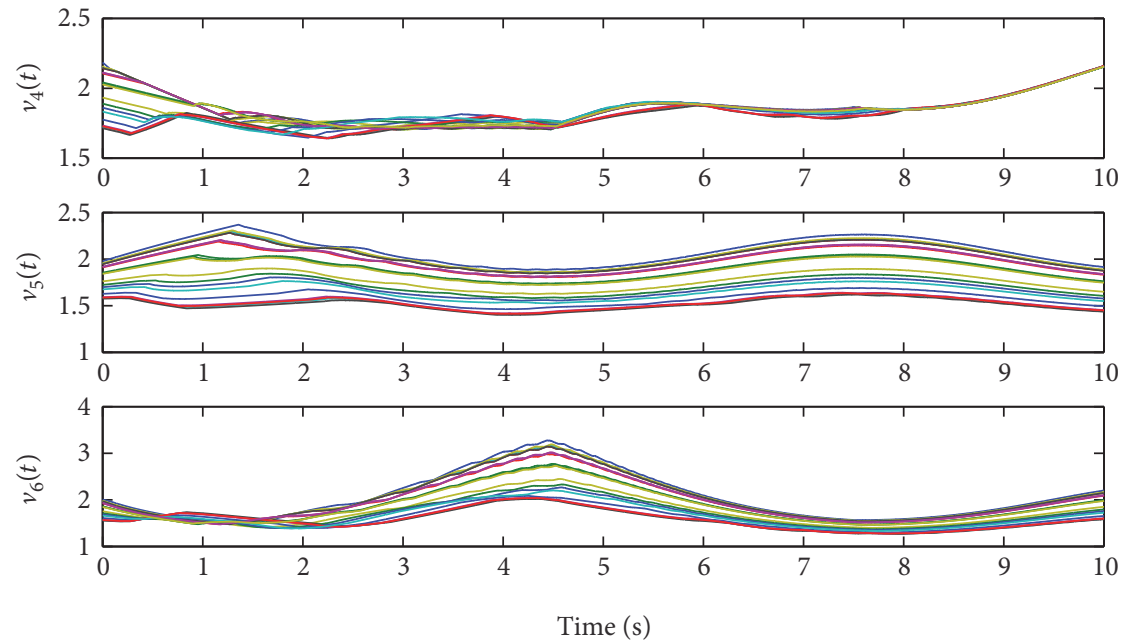

FIGURE 8: Control voltage signals commanded for the last three SJA arrays (i.e., $v_{4}(t), v_{5}(t)$, and $\left.v_{6}(t)\right)$ during closed-loop operation.

$\begin{aligned} \mathbf{A} & =\left[\begin{array}{ccccc}-61.1273 & 0 & -7.6409 \\ 0 & -174.3472 & 0 \\ -7.2692 & 0 & -0.4543\end{array}\right], \\ \mathbf{B} & =\left[\begin{array}{cccccc}-0.2292 & 0.2292 & -0.2292 & 0.2292 & -0.0306 & 0.0306 \\ 0.0599 & 0.0599 & 0.0804 & 0.0804 & -0.0256 & 0.0256 \\ -0.0084 & 0.0084 & -0.535 & 0.0535 & 0.1177 & -0.1177\end{array}\right] .\end{aligned}$

The wind gust model used in the simulation is based on the FAR discrete gust model described in ([33]). The simulation model for the wind gust is based on the expression in (4), see Figure 3.

The results of 20 Monte Carlo-type simulations are shown in Figures 4, 5, 6, 7, and 8. The results were obtained using control gains selected as $\mathbf{k}_{s}=\operatorname{diag}\{0.10,0.15,2.3\}$, $\boldsymbol{\beta}=\operatorname{diag}\{3.3,0.3,0.8\}$, and $\boldsymbol{\gamma}=0.3$. Each set of axes shows the control performance for 20 different scenarios, where
TABLE 1: Constant nominal values of SJA parameters used in simulation.

\begin{tabular}{lllllll}
\hline$\theta_{1}^{*}$ ( deg) & 32.9 & 29.8 & 26.7 & 24.0 & 20.5 & 17.8 \\
$\theta_{2}^{*}$ (volt-deg) & 14.7 & 13.8 & 12.8 & 11.7 & 10.0 & 9.5 \\
\hline
\end{tabular}

each plot shows the closed-loop response in the presence of 20 different sets of off-nominal values for the actual (plant) SJA parameters $\theta_{1 i}^{*}$ and $\theta_{2 i}^{*}$ for $i=1, \ldots, 6$. The 20 sets of parameter values were generated using a randomization routine, which resulted in deviations of the actual SJA parameter values by up to $35.7 \%$ off nominal. The constant estimates (nominal values) used in the simulation are listed in Table 1.

Remark 9 (comparison of results). The capability of the proposed robust nonlinear control method to compensate for SJA parameter deviations of more than 35\% demonstrates a significant improvement over standard adaptive control approaches (cf. [8, 13]). Specifically, the results using 

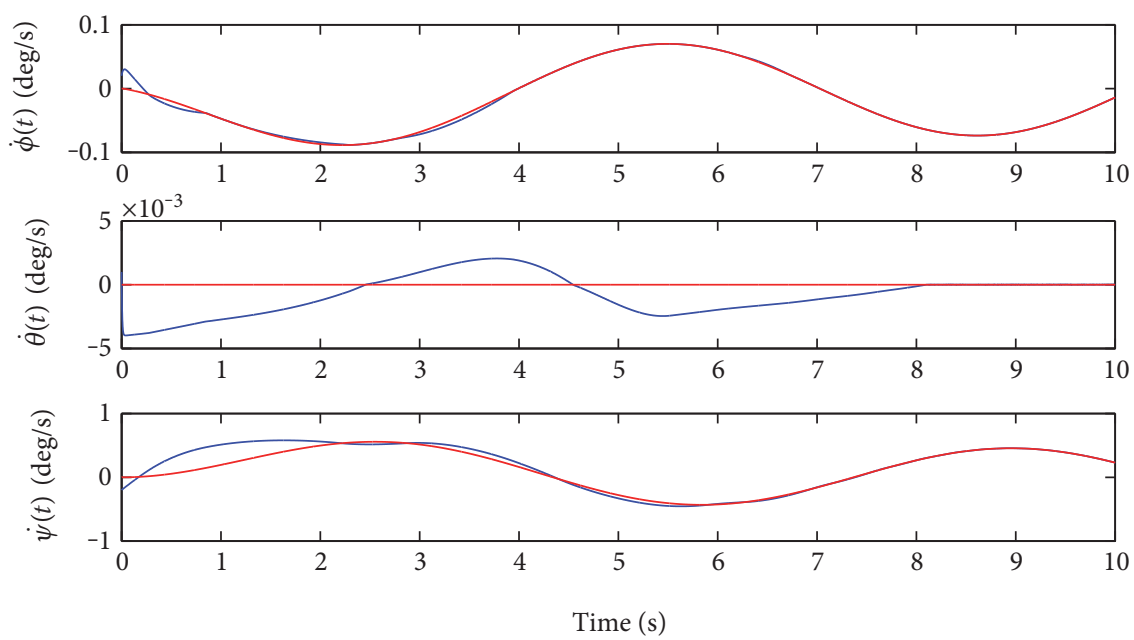

Figure 9: Model reference (red) and actual state (blue) during closed-loop controller operation in the presence of $35.75 \%$ SJA parameter uncertainty.

standard adaptive control approaches assume that the adaptive parameter estimates are within less than $5 \%$ of the actual parameter values.

Figure 4 shows the closed-loop tracking error response and demonstrates rapid convergence of the tracking error to zero in all 20 cases. Figures 5 and 6 show the virtual surface deflection control commands during closed-loop operation, and Figures 7 and 8 show the SJA voltage control inputs commanded during closed-loop operation. The results demonstrate that the closed-loop system remains stable in all 20 cases, and asymptotic tracking is achieved throughout the range of uncertainty tested. Figure 9 shows the convergence of the actual UAV states to the model reference states during closed-loop operation for the first iteration of our Monte Carlo-type simulation. The control commands remain within reasonable limits in all 20 cases.

\section{Conclusion}

A robust nonlinear control method that achieves asymptotic trajectory tracking for a SJA-based aircraft model is presented. The control method is proven to achieve semiglobal asymptotic tracking of a reference trajectory in the presence of SJA actuator parameter uncertainty in addition to external norm-bounded disturbances (i.e., vertical wind gusts). A rigorous stability analysis is carried out to prove that the region of attraction of the closed-loop system can be made arbitrarily large through judicious tuning of a control parameter. The controller is designed to be computationally inexpensive, requiring no function approximators, adaptive laws, or complex computations. By utilizing constant feedforward estimates of the uncertain SJA actuator parameters, a matrix decomposition technique is employed along with a novel error system derivation to compensate for significant SJA parametric uncertainty (i.e., greater than 35\% uncertainty in the SJA parameters). Detailed Monte-Carlo-type numerical simulation results are included to illustrate the effectiveness of the proposed control strategy.

\section{Appendix}

This appendix provides proof of Lemma 2.

Lemma A.1. Provided the sufficient gain condition in (33) is satisfied, the following inequality can be obtained:

$$
\int_{0}^{t} L(\tau) d \tau \leq \beta Q|\mathbf{e}(0)|-\mathbf{e}^{T}(0) \mathbf{N}_{d 1}(0)
$$

Hence, (A.1) can be used to conclude that $P(t) \geq 0$, where $P(t)$ is defined in (35) and (36).

To facilitate the following proof, the expression in (37) will be rewritten in a more advantageous form as follows:

$$
L(t)=\sum_{i=1}^{m}\left(\mathbf{r}_{i}(t)\left(\mathbf{N}_{d 1 i}(t)-\sum_{j=i}^{m} \mathbf{T}_{i j} \boldsymbol{\mu}_{1 j}(t)\right)\right) \in \mathbb{R} .
$$

In (A.2), $\mathbf{r}_{i}(t), \mathbf{N}_{d 1 i}(t), \boldsymbol{\mu}_{1 i}(t) \in \mathbb{R}$ for $i=1, \ldots, m$ denote the ith elements of the vectors $r(t), \mathbf{N}_{d 1}(t)$, and $\boldsymbol{\mu}_{1}(t)$; and $\mathbf{T}_{i j} \in$ $\mathbb{R}$ for $i=1, \ldots, m$ and $j=1, \ldots, m$ denote the $(i, j)$ th elements of the matrix $\mathbf{T}$.

Proof. Integrating both sides of (A.2) yields

$$
\int_{0}^{t} L(\tau) d \tau=\int_{0}^{t} \sum_{i=1}^{m} \mathbf{r}_{i}(\tau)\left(\mathbf{N}_{d 1 i}(\tau)-\sum_{j=i}^{m} \mathbf{T}_{i j} \boldsymbol{\mu}_{1 j}(\tau)\right) d \tau \in \mathbb{R}
$$

Based on the expressions in (8) and (9), the integral in (A.3) can be expressed as 


$$
\begin{aligned}
\int_{0}^{t} L(\tau) d \tau= & \int_{0}^{t} \sum_{i=1}^{m}\left(\dot{\mathbf{e}}_{i}(\tau)\right)\left(\mathbf{N}_{d 1 i}(\tau)-\sum_{j=i}^{m} \mathbf{T}_{i j} \boldsymbol{\mu}_{1 j}(\tau)\right) d \tau \\
& +\int_{0}^{t} \sum_{i=1}^{m}\left(\gamma_{i} \mathbf{e}_{i}(\tau)+\gamma_{i} \eta_{i}(\tau)\right) \\
& \cdot\left(\mathbf{N}_{d 1 i}(\tau)-\sum_{j=i}^{m} \mathbf{T}_{i j} \boldsymbol{\mu}_{1 j}(\tau)\right) d \tau
\end{aligned}
$$

where $\gamma_{i} \in \mathbb{R}$ denotes the $i$ th diagonal element of the control gain matrix $\gamma$. The expression in (A.4) can be rewritten as

$$
\begin{aligned}
\int_{0}^{t} L(\tau) d \tau= & \int_{0}^{t} \sum_{i=1}^{m} \frac{\partial \mathbf{e}_{i}(\tau)}{\partial \tau} \mathbf{N}_{d 1 i}(\tau) d \tau \\
& -\int_{0}^{t} \sum_{i=1}^{m} \frac{\partial \mathbf{e}_{i}(\tau)}{\partial \tau} \sum_{j=i}^{m} \mathbf{T}_{i j} \boldsymbol{\mu}_{1 j}(\tau) d \tau \\
& +\int_{0}^{t} \sum_{i=1}^{m} \gamma_{i} \mathbf{e}_{i}(\tau)\left(\mathbf{N}_{d 1 i}(\tau)-\sum_{j=i}^{m} \mathbf{T}_{i j} \boldsymbol{\mu}_{1 j}(\tau)\right) d \tau
\end{aligned}
$$

By evaluating the first integral in (A.5) using integration by parts, (A.5) can be expressed as

$$
\begin{aligned}
\int_{0}^{t} L(\tau) d \tau= & \sum_{i=1}^{m} \mathbf{e}_{i}(t) \mathbf{N}_{d 1 i}(t)-\sum_{i=1}^{m} \mathbf{e}_{i}(0) \mathbf{N}_{d 1 i}(0) \\
& -\int_{0}^{t} \sum_{i=1}^{m} \mathbf{e}_{i}(\tau) \frac{\partial \mathbf{N}_{d 1 i}(\tau)}{\partial \tau} d \tau \\
& -\int_{0}^{t} \sum_{i=1}^{m} \frac{\partial \mathbf{e}_{i}(\tau)}{\partial \tau} \sum_{j=i}^{m} \mathbf{T}_{i j} \boldsymbol{\mu}_{1 j}(\tau) d \tau \\
& +\int_{o}^{t} \sum_{i=1}^{m} \gamma_{i} \mathbf{e}_{i}(\tau)\left(\mathbf{N}_{d 1 i}(\tau)-\sum_{j=i}^{m} \mathbf{T}_{i j} \boldsymbol{\mu}_{1 j}(\tau)\right) d \tau
\end{aligned}
$$

After substituting the definition of the auxiliary control term $\mu_{1}(t)$ given in (16) and rearranging, (A.6) can be expressed as

$$
\begin{array}{r}
\int_{0}^{t} L(\tau) d \tau=\sum_{i=1}^{m}\left(\mathbf{e}_{i}(t) \mathbf{N}_{d 1 i}(t)-\mathbf{e}_{i}(0) \mathbf{N}_{d 1 i}(0)\right. \\
\left.-\int_{0}^{t} \frac{\partial \mathbf{e}_{i}(\tau)}{\partial \tau} \sum_{j=i}^{m} \mathbf{T}_{i j} \beta \operatorname{sgn}\left(\gamma_{j} \mathbf{e}_{j}(\tau)\right) d \tau\right) \\
+\int_{0}^{t} \sum_{i=1}^{m} \gamma_{i} \mathbf{e}_{i}(\tau)\left(\mathbf{N}_{d 1 i}(\tau)-\frac{1}{\gamma} \frac{\partial \mathbf{N}_{d 1 i}(\tau)}{\partial \tau}\right. \\
\left.-\sum_{j=i}^{m} \mathbf{T}_{i j} \beta \operatorname{sgn}\left(\gamma_{j} \mathbf{e}_{j}(\tau)\right)\right) d \tau .
\end{array}
$$

By using the fact that

$$
\sum_{j=i}^{m} \mathbf{T}_{i j} \beta \operatorname{sgn}\left(\boldsymbol{\gamma}_{j} \mathbf{e}_{j}(\tau)\right)=\boldsymbol{\beta}\left(\operatorname{sgn}\left(\boldsymbol{\gamma}_{j} \mathbf{e}_{j}(\tau)\right)+\sum_{j=i+1}^{m} \overline{\mathbf{T}}_{i j} \operatorname{sgn}\left(\mathbf{e}_{j}(\tau)\right)\right),
$$

the bounding inequalities in (21) can be used to express (A.7) as

$$
\begin{aligned}
\int_{0}^{t} L(\tau) d \tau= & \sum_{i=1}^{m}\left(\mathbf{e}_{i}(t) \mathbf{N}_{d 1 i}(t)-\mathbf{e}_{i}(0) \mathbf{N}_{d 1 i}(0)\right) \\
& -\int_{0}^{t} \sum_{i=1}^{m} \frac{\partial \mathbf{e}_{i}(\tau)}{\partial \tau} \boldsymbol{\beta} \delta \operatorname{sgn}\left(\gamma_{i} \mathbf{e}_{i}(\tau)\right) d \tau+\int_{0}^{t} \sum_{i=1}^{m} \gamma_{i} \mathbf{e}_{i}(\tau) \\
& \cdot\left(\mathbf{N}_{d 1 i}(\tau)-\frac{1}{\gamma} \frac{\partial \mathbf{N}_{d 1 i}(\tau)}{\partial \tau}-\delta \boldsymbol{\beta} \operatorname{sgn}\left(\gamma_{i} \mathbf{e}_{i}(\tau)\right)\right) d \tau
\end{aligned}
$$

where $\delta \in(\varepsilon, Q)$ is a positive constant parameter. By using the property

$$
\int_{0}^{t} \frac{\partial \mathbf{e}(\tau)}{\partial \tau} \operatorname{sgn}(\gamma \mathbf{e}(\tau)) d \tau=|\mathbf{e}(t)|-|\mathbf{e}(0)|,
$$

the expression in (A.9) can be rewritten as

$$
\begin{aligned}
\int_{0}^{t} L(\tau) d \tau= & -\sum_{i=1}^{m} \mathbf{e}_{i}(0) \mathbf{N}_{d 1 i}(0)+\sum_{i=1}^{m} \boldsymbol{\beta} \delta\left|\mathbf{e}_{i}(0)\right| \\
& +\sum_{i=1}^{m} \mathbf{e}_{i}(t) \mathbf{N}_{d 1 i}(t)-\sum_{i=1}^{m} \boldsymbol{\beta} \delta\left|\mathbf{e}_{i}(t)\right|+\int_{0}^{t} \sum_{i=1}^{m} \gamma_{i} \mathbf{e}_{i}(\tau) \\
& \cdot\left(\mathbf{N}_{d 1 i}(\tau)-\frac{1}{\gamma} \frac{\partial \mathbf{N}_{d 1 i}(\tau)}{\partial \tau}-\delta \boldsymbol{\beta} \operatorname{sgn}\left(\gamma_{i} \mathbf{e}_{i}(\tau)\right)\right) d \tau
\end{aligned}
$$

The expression in (A.11) can be upper bounded as

$$
\begin{aligned}
\int_{0}^{t} L(\tau) d \tau \leq & -\mathbf{e}^{T}(0) \mathbf{N}_{d 1}(0)+\beta Q|\mathbf{e}(0)|+\sum_{i=1}^{m}\left(\zeta_{\mathbf{N}_{d 1}}-\varepsilon \boldsymbol{\beta}\right)\left|\mathbf{e}_{i}(t)\right| \\
& +\int_{0}^{t} \sum_{i=1}^{m} \gamma_{i}\left|\mathbf{e}_{i}(\tau)\right|\left(\zeta_{\mathbf{N}_{d 1}}-\frac{1}{\gamma} \zeta_{\dot{\mathbf{N}}_{d 1}}-\varepsilon \boldsymbol{\beta}\right) d \tau
\end{aligned}
$$

Thus, it is clear from (A.12) that if $\boldsymbol{\beta}$ satisfies the sufficient condition in (33), then

$$
\int_{0}^{t} L(\tau) d \tau \leq \beta Q|\mathbf{e}(0)|-\mathbf{e}(0)^{T} \mathbf{N}_{d 1}(0) .
$$

Hence, $P(t) \geq 0$ from (35), (36), and (A.13).

\section{Conflicts of Interest}

The authors declare that they have no conflicts of interest.

\section{Acknowledgments}

This research is supported in part by National Science Foundation Award no. 1335405. 


\section{References}

[1] S. Bhatt, V. V. Golubev, and Y. Tang, "Design, modeling and testing of synthetic jet actuators for MAV flight control," in 52nd Aerospace Sciences Meeting, pp. 1-17, National Harbor, MD, USA, January 2014.

[2] M. Gul, O. Uzol, and I. S. Akmnador, "Flow control with synthetic jet actuators under adverse pressure gradient laminar boundary layer," in 32nd AIAA Applied Aerodynamics Conference, pp. 1-17, Atlanta, GA, USA, June 2014.

[3] M. G. De Giorgi, C. G. De Luca, A. Ficarella, and F. Marra, "Comparison between synthetic jets and continuous jets for active flow control: application on a NACA 0015 and a compressor stator cascade," Aerospace Science and Technology, vol. 43, pp. 256-280, 2015.

[4] H. Tang, P. Salunkhe, Y. Zheng, J. Du, and Y. Wu, "On the use of synthetic jet actuator arrays for active flow separation control," Experimental Thermal and Fluid Science, vol. 57, pp. 1-10, 2014.

[5] V. Golubev, P. Kazarin, W. MacKunis, S. Borener, and D. Hufty, "Analysis of safety implications for SJA-based robust UAS flight control technology," in 2015 IEEE/AIAA 34th Digital Avionics Systems Conference (DASC), pp. 1-31, Prague, Czech Republic, 2015.

[6] H. E. Monir, M. Tadjfar, and A. Bakhtian, "Tangential synthetic jets for separation control," Journal of Fluids and Structures, vol. 45, pp. 50-65, 2013.

[7] M. Amitay, D. R. Smith, V. Kibens, D. E. Parekh, and A. Glezer, "Aerodynamic flow control over an unconventional airfoil using synthetic jet actuators," AIAA Journal, vol. 39, no. 3, pp. 361-370, 2001.

[8] D. Deb, G. Tao, J. O. Burkholder, and D. R. Smith, “Adaptive compensation control of synthetic jet actuator arrays for airfoil virtual shaping," Journal of Aircraft, vol. 44, no. 2, pp. 616-626, 2007.

[9] W. MacKunis, S. Subramanian, S. Mehta, J. W. Curtis, M. Reyhanoglu, and C. Ton, "Robust nonlinear aircraft tracking control using synthetic jet actuators," in 52nd IEEE Conference on Decision and Control, pp. 220-225, Florence, Italy, December 2013.

[10] D. Deb, G. Tao, J. O. Burkholder, and D. R. Smith, "An adaptive inverse control scheme for a synthetic jet actuator model," in Proceedings of the 2005, American Control Conference, 2005, pp. 2646-2651, Portland, OR, USA, June 2005.

[11] D. Deb, G. Tao, J. O. Burkholder, and D. R. Smith, “An adaptive inverse control scheme for synthetic jet actuator arrays," in Infotech@Aerospace, Arlington, VA, USA, September 2005.

[12] D. Deb, G. Tao, J. O. Burkholder, and D. R. Smith, "Adaptive synthetic jet actuator compensation for a nonlinear tailless aircraft model at low angles of attack," in 2006 American Control Conference, p. 6, Minneapolis, MN, USA, June 2006.

[13] D. Deb, G. Tao, J. O. Burkholder, and D. R. Smith, "Adaptive synthetic jet actuator compensation for a nonlinear aircraft model at low angles of attack," IEEE Transactions on Control Systems Technology, vol. 16, no. 5, pp. 983-995, 2008.

[14] G. Hong, "Numerical investigation to forcing frequency and amplitude of synthetic jet actuators," AIAA Journal, vol. 50, no. 4, pp. 788-796, 2012.

[15] M. Kim, S. Kim, W. Kim, C. Kim, and Y. Kim, "Flow control of tiltrotor unmanned-aerial-vehicle airfoils using synthetic jets," Journal of Aircraft, vol. 48, no. 3, 2011.
[16] W. Kim, C. Kim, and K. J. Jung, "Separation control characteristics of synthetic jets depending on exit configuration," AIAA Journal, vol. 50, no. 3, 2012.

[17] R. B. Kotapati, R. Mittal, O. Marxen, F. Ham, D. You, and L. N. Cattafesta III, "Nonlinear dynamics and synthetic-jet-based control of a canonical separated flow," Journal of Fluid Mechanics, vol. 654, pp. 65-97, 2010.

[18] A. Kourta and C. Leclerc, "Characterization of synthetic jet actuation with application to Ahmed body wake," Sensors and Actuators A: Physical, vol. 192, pp. 13-26, 2013.

[19] Y. Liu, M. Ciuryla, M. Amitay et al., "Integrated flight control and flow control using synthetic jet arrays," in AIAA Guidance, Navigation, and Control Conference and Exhibit, Keystone, CO, USA, 2006.

[20] O. D. L. Mejia, R. D. Moser, D. P. Brzozowski, and A. Glezer, "Effects of trailing-edge synthetic jet actuation on an airfoil," AIAA Journal, vol. 49, no. 8, 2011.

[21] Z. B. Luo, Z. X. Xia, and Y. G. Xie, "Jet vectoring control using a novel synthetic jet actuator," Chinese Journal of Aeronautics, vol. 20, no. 3, pp. 193-201, 2007.

[22] T. Ozawa, S. Lesbros, and G. Hong, "LES of synthetic jets in boundary layer with laminar separation caused by adverse pressure gradient," Computers and Fluids, vol. 39, no. 5, pp. 845-858, 2010.

[23] D. A. Tamburello and M. Amitay, "Active control of a free jet using a synthetic jet," International Journal of Heat and Fluid Flow, vol. 29, no. 4, pp. 967-984, 2008.

[24] S. Tardu, "Forcing a low Reynolds number channel flow to generate synthetic turbulent-like structures," Computers \& Fluids, vol. 55, pp. 101-108, 2012.

[25] Z. Travnicek, L. Nemcova, J. Kordik, V. Tesar, and V. Kopecky, "Axisymmetric impinging jet excited by a synthetic jet system," International Journal of Heat and Mass Transfer, vol. 55, no. 4, pp. 1279-1290, 2012.

[26] L.-Y. Tseng, A.-S. Yang, and J.-C. Lin, "Study of a crossflow over a zero-net-mass-flux synthetic jet driven by a vibrating diaphragm," Journal of Mechanics, vol. 27, no. 4, pp. 503510, 2011.

[27] B. Vukasinovic, A. Glezer, S. Gordeyev, E. Jumper, and V. Kibens, "Fluidic control of a turret wake, part I: aerodynamic effects," in 47th AIAA Aerospace Sciences Meeting Including The New Horizons Forum and Aerospace Exposition, Orlando, FL, USA, 2009.

[28] S. Zhang and S. Zhong, "Experimental investigation of flow separation control using an array of synthetic jets," AIAA Journal, vol. 48, no. 3, pp. 611-623, 2010.

[29] S. T. Mondschein, G. Tao, and J. O. Burkholder, "Adaptive actuator nonlinearity compensation and disturbance rejection with an aircraft application," in Proceedings of the 2011 American Control Conference, pp. 2951-2956, San Francisco, CA, USA, 2011.

[30] R. Nelson, Flight Stability and Automatic Control, McGrawHill, 2nd edition, 1998.

[31] A. A. Tchieu, A. T. Kutay, J. A. Muse, A. J. Calise, and A. Leonard, "Validation of a low-order model for closed loop flow control enabled flight," in 4th Flow Control Conference, Seattle, WA, USA, 2008.

[32] C. Singhal, G. Tao, and J. O. Burkholder, "Neural networkbased compensation of synthetic jet actuator nonlinearities for aircraft flight control," in AIAA Guidance, Navigation, and Control Conference, Chicago, IL, USA, 2009. 
[33] "Airworthiness standards: transport category airplanes," in Federal Aviation Regulations, Department of Transportation, Washington, DC, USA, 2015.

[34] A. S. Morse, "A gain matrix decomposition and some of its applications," Systems \& Control Letters, vol. 21, no. 1, pp. 1-10, 1993.

[35] H. K. Khalil, Nonlinear Systems, Prentice Hall, Upper Saddle River, NJ, USA, 3rd edition, 2002. 


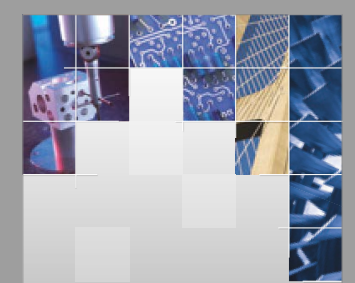

\section{Enfincering}
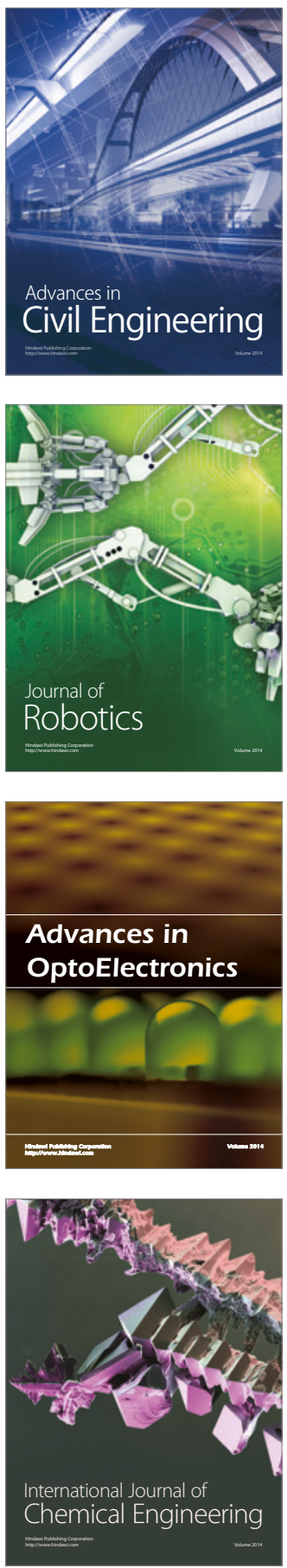

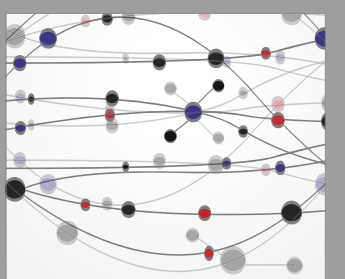

The Scientific World Journal

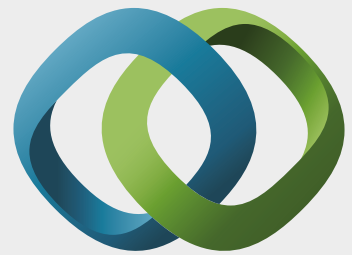

\section{Hindawi}

Submit your manuscripts at

https://www.hindawi.com
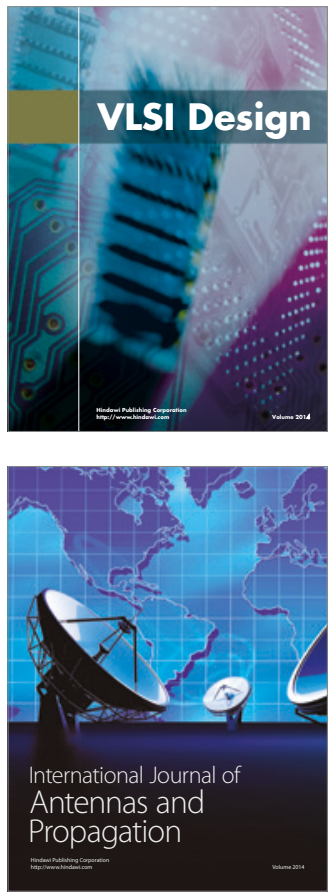

\section{Rotating}

Machinery
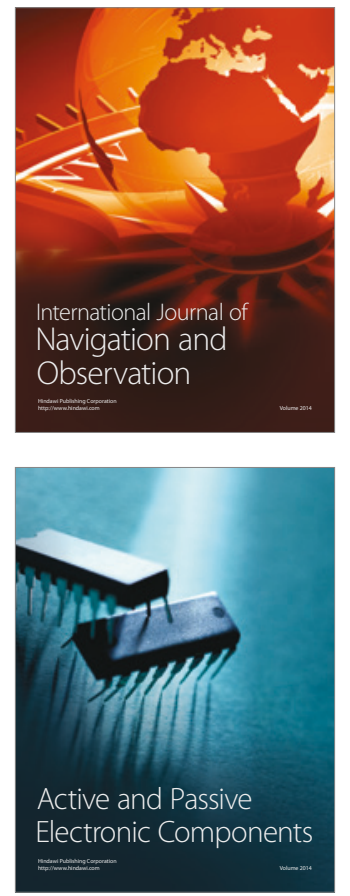
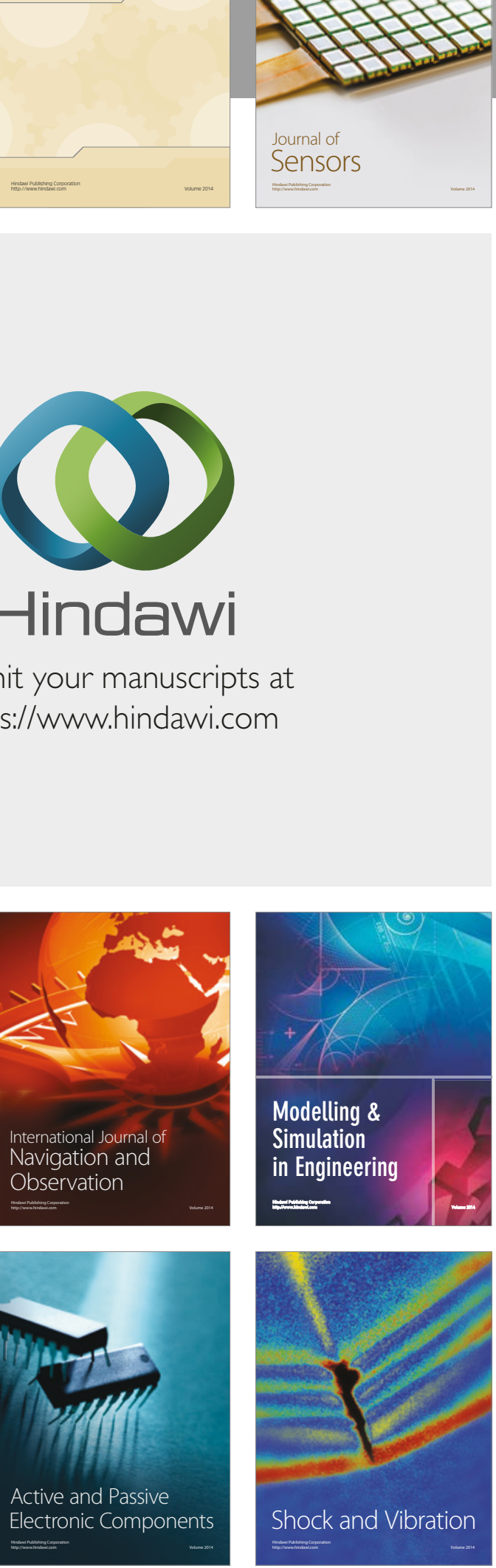
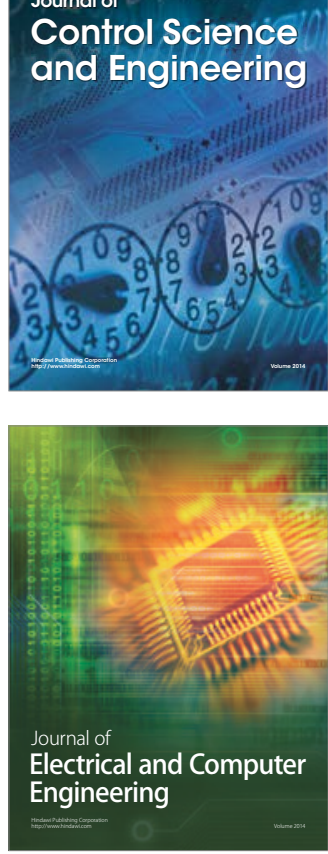

Distributed

Journal of

Control Science

and Engineering
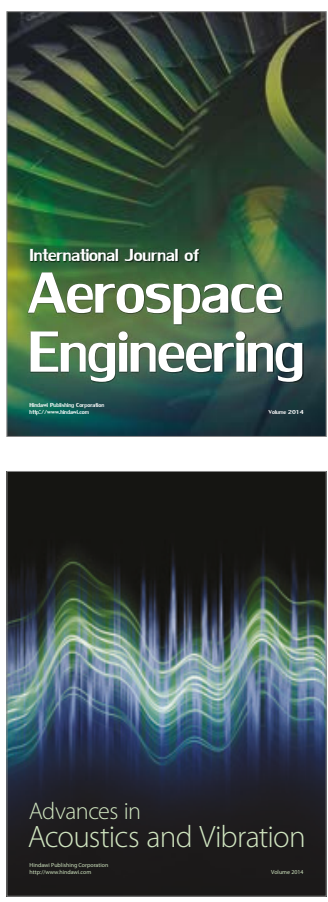

Sensor Networks 